12

\title{
Электростатический спектрограф с большим диапазоном одновременно регистрируемых энергий из двух коаксиальных электродов с закрытыми торцами и дискретным комбинированным внешним электродом
}

\author{
(C) Т.Я. Фишкова \\ Физико-технический институт им. А.Ф. Иофффе РАН, \\ 194021 Санкт-Петербург, Россия \\ e-mail: fishkova@mail.ru
}

(Поступило в Редакцию 7 июня 2017 г.)

\begin{abstract}
Путем компьютерного моделирования найден оптимальный вариант геометрических и электрических параметров электростатического спектрографа заряженных частиц высокой светосилы с диапазоном одновременно регистрируемых энергий $E / E_{\min }=1-50$, что особенно важно для анализа заряженных частиц по энергии при быстропротекающих процессах в различного рода веществах. Спектрограф состоит из двух коаксиальных электродов с закрытыми с помощью плоских электродов торцами. Внешний электрод, имеющий конусно-цилиндрическую форму, разрезан на части с потенциалами, возрастающими по линейному закону, за исключением последней цилиндрической части, которая электрически соединена с задним торцевым электродом. Внутренний цилиндрический электрод, а также передний торцевой электрод заземлены. Во всем диапазоне энергий система обладает острой фокусировкой на внутренний цилиндрический электрод, что обеспечивает разрешение по энергии не хуже $3 \cdot 10^{-3}$.
\end{abstract}

DOI: $10.21883 /$ JTF.2018.01.45494.2373

\section{Введение}

В работе [1] предложен энергоанализатор в виде цилиндрического конденсатора с разрезанным на части внешним электродом, потенциал на котором меняется по линейному закону, что позволяет параллельно (одновременно) регистрировать пучки заряженных частиц различных энергий. Однако такая система мало пригодна для практического использования из-за открытых торцов цилиндрического конденсатора, способствующих краевым эффектам, которые не учитываются при расчетах. Кроме того, возникают сомнения в возможности расположения исследуемого объекта (источник вторичных электронов), а также источника первичных электронов либо других видов радиоизлучений внутри цилиндрического электрода меньшего диаметра, не ясна также и конструкция многоканального детектора для одновременной регистрации пучков различных энергий.

В работе [2] предложен и исследован модифицированный энергоанализатор на основе цилиндрического конденсатора с плоскими торцевыми электродами, закрепленными на внутреннем заземленном цилиндрическом электроде, что упрощает питание системы, а главное, исключает краевые эффекты. В отличие от спектрографа [1] объект и системы формирования первичного излучения вынесены за пределы поля. Разработана также схема одновременного детектирования вторичных частиц различных энергий. Недостатком системы [2] является то обстоятельство, что во всем диапазоне регистрируемых энергий классическая фокусировка имеет место при несколько отличающихся параметрах на входе в систему.
Положение объекта исследования и величины углов впуска пучков заряженных частиц различных энергий, обеспечивающие острую фокусировку, не одинаковы. Поэтому величины разрешения такого спектрографа во всем диапазоне энергий довольно сильно отличаются друг от друга, что не обеспечивает эффективного использования спектрографа [2] для решения задач при быстропротекающих процессах.

Целью настоящей работы является разработка простой конструкции светосильного спектрографа по энергии и его питания, при которых одновременно осуществляется острая фокусировка пучков заряженных частиц различных энергий. При этом обеспечивается высокое разрешение во всем диапазоне регистрируемых энергий, что необходимо для эффективного анализа состава разного рода веществ, в том числе биологических объектов.

\section{Компьютерное моделирование}

Задача о нахождении электронно-оптических свойств предлагаемого светосильного спектрографа решается по программе TEO, написанной ранее с участием автора настоящей работы, для расчета различного рода полей и траекторий заряженных частиц в двумерных электростатических системах. В настоящей работе исследовано несколько вариантов простых двухэлектродных осесимметричных конструкций электростатических систем с закрытыми торцами, имеющих одинаковые габаритные размеры. Причем один из электродов исследуемых спектрографов разрезан на части, что позволяет одновременно регистрировать в широком диапазоне энергии пучков 
заряженных частиц. Путем компьютерного моделирования рассмотрены следующие конструкции коаксиальных двухэлектродных электростатических спектрографов с вынесенным за пределы поля объектом исследования и детектором в виде плоских электродов, установленных за выходными щелями перпендикулярно продольной оси системы [2]: 1) оба электрода имеют конусную форму с величиной угла полураствора, меняющегося в пределах $\beta=\left(5^{\circ}-20^{\circ}\right)$, при этом внешний электрод разрезан на части; 2) внешний разрезной электрод выполнен в виде конуса с теми же углами раствора,что в 1), внутренний электрод выполнен в виде цилиндра; 3) внешний разрезной электрод выполнен как комбинация последовательно расположенных цилиндрического и конусного сегментов в виде раструба, внутренний электрод выполнен в виде цилиндра; 4) внешний разрезной электрод является комбинацией последовательно расположенных конусного и цилиндрического сегментов с различным количеством частей, внутренний электрод по-прежнему является сплошным цилиндрическим.

Питание на частях внешнего разрезного электрода осуществлялось тремя различными способами: по линейному закону, а также по законам квадратного корня, либо корня четвертой степени, поскольку автором ранее показано, что для спектрографов с плоскими электродами с уменьшением роста потенциалов на частях разрезного электрода растет диапазон одновременно регистрируемых энергий. Наилучшим оказалось питание разрезного комбинированного электрода, когда распределение потенциалов на его частях возрастает по линейному закону, как в работе [1].

Компьютерное моделирование указанных выше систем в диапазоне изменения энергий заряженных частиц, равном $E / E_{\min }=1-50$, показало, что с точки зрения одинаковых фокусирующих свойств в указанном диапазоне энергий оптимальной является конструкция в соответствии с четвертым вариантом. Поиски геометрии такой системы с точки зрения наилучшей фокусировки проводились по следующим независимым параметрам: углу полураствора конусного электрода $\beta=\left(5^{\circ}-20^{\circ}\right)$, начальному малому радиусу конуса $R_{b}=(2.5-5) R$, где $R$ - радиус внутреннего цилиндрического электрода, а также трем связанным между собой параметрам - по количеству частей разрезного электрода $n=(10-15)$, длине отдельных частей $l_{n}=(1-2) R$ и расстоянию между ними $s_{n}=(0.5-0.25) R$. Расчеты показали, что оптимальной оказалась следующая геометрия спектрографа: угол полураствора конусного электрода $\beta=10^{\circ}$, величина малого радиуса конусного электрода $R_{b}=5 R$, а радиус цилиндрического сегмента внешнего электрода равен $R_{a}=7.5 R$. Длина частей разрезного электрода $l_{n}=1.5 R$, расстояние между ними $s_{n}=0.25 R$. При неизменной общей длине анализатора $L=25 R$ оптимальным оказалось разбиение внешнего электрода на $n=14$ равных частей при количестве частей конусного сегмента $n_{c}=8$, а цилиндрического сегмента $-n_{c}=6$. В этом случае длина конусного сегмента $H$ равна $0.55 L$.

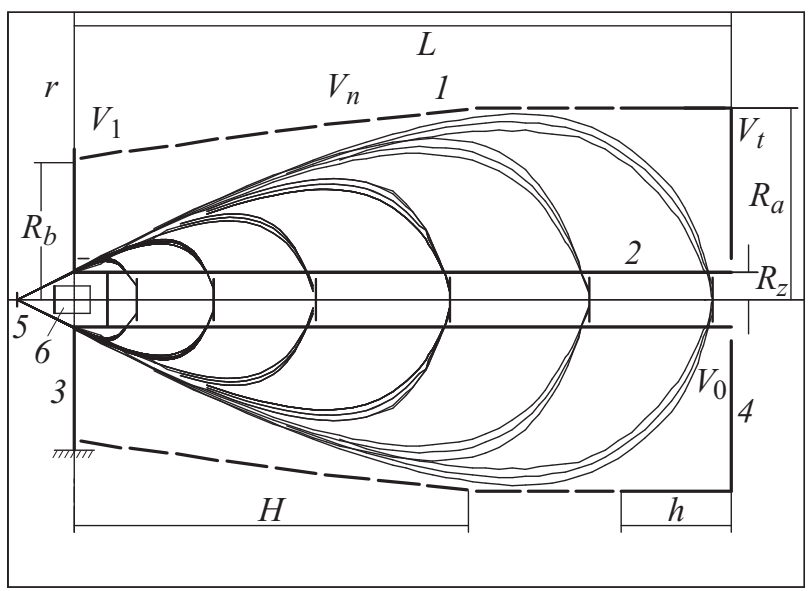

Схема светосильного спектрографа из двух коаксиальных электродов с закрытыми торцами и ход траекторий пучков заряженных частиц с энергиями, последовательно равными $E / E_{\min }=1,5,12.5,25,37.5,50.1$ - внешний разрезной конусно-цилиндрический электрод, 2 - внутренний цилиндрический электрод, 3 - передний плоский торцевой электрод, 4 - задний плоский торцевой электрод, 5- исследуемый объект, 6 - источник первичного излучения.

Последние три участка цилиндрического сегмента электрически соединены между собой и с задним плоским торцевым электродом. Такая система длиной $h=5.5 R$ способствует улучшению фокусировки пучков высоких энергий, проходящих вблизи задней стенки анализатора.

Величины потенциалов на $(n-1)$ частях комбинированного электрода задаются по эмпирической формуле автора, т.е. иначе, чем в работе [1]. При заземленных внутреннем цилиндрическом электроде и переднем плоском торцевом электроде потенциалы на частях внешнего комбинированного электрода равны

$$
V_{n}=V_{1}\left(z_{n} / z_{1}\right), z_{n}=z_{1}[1+1.75(n-1)],
$$

где $V_{1}$ - потенциал на первой части разрезного электрода, $n-$ порядковый номер соответствующей части, отсчитываемый от входа в систему, $z_{n}$ - расстояния от передней стенки до середины соответствующей $(n-1)$ части конусно-цилиндрического электрода, $z_{1}=R$. Потенциал $V_{1}$, по которому определяются питающие напряжения на последующих частях разрезного электрода в соответствии с формулой (1), связан с силой спектрографа на первом участке следующим образом: $F=e V_{1} / E_{\min }(e-$ заряд частицы). Оптимальная сила $F$ находилась, исходя из требования, чтобы пучок наилучшим образом вписывался в апертуру спектрографа и фокусировался на внутренний цилиндрический электрод.

Ранее в работе автора [2] установлено, что впуск пучков заряженных частиц следует производить через заземленный торцевой электрод вблизи продольной оси системы, поэтому координата $z_{i}$ исследуемого объекта, выведенного за пределы системы и расположенного на продольной оси анализатора $r_{i}=0$, менялась в пределах $z_{i}=-(2-2.5) R$ при величине начальных углов 
Таблица 1. Основные параметры предлагаемого спектрографа при углах раствора пучков различных энергий, равных $\delta \theta=1^{\circ}$

\begin{tabular}{c|c|c|c|c|c|c|c|c|c|c|c}
\hline$E / E_{\min }$ & 1 & 2.5 & 5 & 7.5 & 10 & 15 & 25 & 30 & 40 & 45 & 50 \\
\hline$z_{f} / R$ & 1.80 & 3.08 & 4.70 & 6.09 & 7.37 & 9.74 & 14.1 & 16.1 & 20.1 & 21.9 & 23.9 \\
$D / R$ & 1.0 & 1.80 & 2.95 & 4.1 & 5.0 & 6.8 & 10.0 & 12.0 & 14.8 & 15.8 & 21.0 \\
$\rho \cdot 10^{-3}$ & 10 & 8.6 & 5.3 & 4.4 & 2.6 & 2.4 & 3.1 & 3.0 & 2.5 & 2.2 & 3.1
\end{tabular}

Таблица 2. Разрешение спектрографа на низких энергиях при углах раствора пучков $\delta \theta=0.8^{\circ}$

\begin{tabular}{c|c|c|c|c}
\hline$E / E_{\min }$ & 1 & 2.5 & 5 & 7.5 \\
\hline$\rho \cdot 10^{-3}$ & 5.5 & 3.1 & 2.9 & 2.2
\end{tabular}

наклона пучков к продольной оси системы $\theta$ в диапазоне $\theta=\left(22^{\circ}-32^{\circ}\right)$. Численные расчеты по программе TEO показали, что наиболее острая фокусировка пучков различных энергий в диапазоне $E / E_{\min }=1-50$ на внутренний цилиндрический электрод происходит при $z_{i}=-2.15 R$ и $\theta=(26.6 \pm 0.5)^{\circ}$.

Схематическое устройство спектрографа оптимальной геометрии из двух коаксиальных электродов с комбинированным внешним и цилиндрическим внутренним электродами с закрытыми торцами, а также ход траекторий пучков заряженных частиц в таком спектрографе представлены на рисунке. При этом исследуемый объект (источник вторичных электронов), а также источник первичного излучения вынесены за пределы поля. Детектор состоит из ряда плоских электродов, установленных внутри цилиндрического электрода перпендикулярно продольной оси спектрографа.

Питание спектрографа осуществлялось следующим образом: потенциал на первой части разрезного электрода равен $V_{1}=\left[2.2 E_{\min } / e\right]$, потенциалы на последующих семи частях конусного и трех частях цилиндрического сегментов внешнего электрода задаются по линейному закону согласно формуле (1). Последние три части цилиндрического сегмента, соединенные электрически с задней торцевой стенкой, имеют оптимизированный с точки зрения наилучшей фокусировки пучков высоких энергий потенциал $V_{t}$, равный $19.5 V_{1}$. Внутренний цилиндрический электрод вместе с передней торцевой стенкой заземлен.

Основные параметры предлагаемого спектрографа с указанной выше оптимальной геометрией и питанием в диапазоне параллельно регистрируемых энергий, равном $E / E_{\min }=1-50$, приведены в табл. 1. В таблице введены следующие обозначения: $z_{f}-$ положение точек фокусировки центральных траекторий пучков различных энергий на внутренний цилиндрический электрод, $D$ - коэффициенты линейной дисперсии по энергии, $\rho=\delta E / E$ - разрешение по энергии при угле раствора пучка $\delta \theta=1^{\circ}$.
Из табл. 1 видно, что при средних и высоких энергиях разрешение не хуже величины $\rho=3 \cdot 10^{-3}$, при низких энергиях оно в $1.5-2$ раза хуже. Поскольку желательно во всем диапазоне регистрируемых энергий иметь примерно одинаковое разрешение по энергии, можно сузить выходные щели во внутреннем цилиндрическом электроде, несколько уменьшив угол раствора пучка. В табл. 2 приведены величины разрешения спектрографа по энергии в диапазоне низких энергий $E=(1-7.5) E_{\min }$ при угле раствора пучков заряженных частиц $\delta \theta=0.8^{\circ}$. При этом светосила спектрографа уменьшается в 1.25 раз, однако разрешение по энергии улучшается в 2 раза и составляет среднюю величину по диапазону $E=(10-50) E_{\min }$, примерно равную $3 \cdot 10^{-3}$. Дальнейшее уменьшение угла раствора пучка до $\delta \theta=0.6^{\circ}$ позволяет при минимальной энергии достичь $\rho=2.8 \cdot 10^{-3}$.

В системах с осевой симметрией светосила (величина телесного угла $\Omega$, исходящего из центра источника заряженных частиц, в процентах от $4 \pi$ ) определяется по известной формуле $\Omega=\alpha \sin \theta_{0} \cdot 100 \%$, где $\alpha-$ угол полураствора пучка в радианах, $\theta_{0}-$ начальный угол наклона центральной траектории пучка к продольной оси системы. В найденном оптимальном режиме работы спектрографа при угле раствора пучка $\delta \theta=1.0^{\circ}$ светосила составляет $\Omega=0.39 \%$. За счет уменьшения угла раствора пучка светосила спектрографа несколько падает до $\Omega=(0.31-0.23) \%$. Следует отметить, что эта величина в 30-40 раз превышает светосилу спектрографов, состоящих из плоских электродов.

\section{Заключение}

С целью изучения состава различного рода веществ при быстропротекающих процессах предложено и исследовано несколько вариантов энергоанализаторов из двух коаксиальных электродов с закрытыми торцами при различного рода простых конструкциях внешнего разрезанного на части электрода, что позволяет осуществлять одновременную регистрацию пучков заряженных частиц в широком диапазоне энергий. Методом компьютерного моделирования найдена оптимальная конструкция разрезного внешнего электрода спектрографа в виде последовательно расположенных конусного и цилиндрического сегментов, подключенных к источнику питания с возможностью подачи на их части потенциалов, возрастающих по линейному закону. При этом внутренний цилиндрический электрод вместе с плоским торцевым 
электродом на входе заземлены. Задний плоский торцевой электрод соединен с последней частью цилиндрического сегмента и подключен к другому источнику питания.

Определен оптимальный режим работы, при котором в диапазоне энергий, различающихся в 50 раз, осуществляется острая фокусировка пучков на внутренний цилиндрический электрод с последующей регистрацией многоканальным измерительным устройством. Для средних и больших энергий разрешение по энергии предлагаемого спектрографа достигает $\rho=3 \cdot 10^{-3}$, для малых энергий $\rho=(5-10) \cdot 10^{-3}$. При этом его светосила достигает величины $0.39 \%$ от $4 \pi$. При исследовании состава тех или иных веществ предлагаемый спектрограф за счет большой светосилы обладает высокой чувствительностью.

\section{Список литературы}

[1] Read F.H. // Rev. Sci. Instr. 2002. Vol. 73. N 3. P. 1129-1139.

[2] Фишкова Т.Я. // ЖТФ. 2016. Т. 86. Вып. 9. С. 153-155. 